\title{
Germanica
}

\section{Le drame brûle-t-il ? Manhattan Medea de Dea Loher}

Manhattan Medea, by Dea Loher. Is Drama Going Up In Flames?

Geht das Drama in Flammen auf? Manhattan Medea von Dea Loher

\section{Francine Maier-Schaeffer}

\section{OpenEdition}

Journals

Édition électronique

URL : http://journals.openedition.org/germanica/2592

DOI : 10.4000/germanica.2592

ISSN : 2107-0784

\section{Éditeur}

Université de Lille

\section{Édition imprimée}

Date de publication : 30 juin 2014

Pagination : $125-140$

ISBN : 9782913857339

ISSN : 0984-2632

\section{Référence électronique}

Francine Maier-Schaeffer, «Le drame brûle-t-il ? Manhattan Medea de Dea Loher », Germanica [En ligne], 54 | 2014, mis en ligne le 30 juin 2017, consulté le 06 octobre 2020. URL : http://

journals.openedition.org/germanica/2592; DOI : https://doi.org/10.4000/germanica.2592

\section{(c) Tous droits réservés}




\title{
Le drame brûle-t-il ? Manhattan Medea de Dea Loher
}

\author{
Francine MAIER-SCHAEFFER \\ Université de Haute-Bretagne - Rennes 2
}

« Pourquoi le théâtre devrait-il s'allier à ses auteurs ? Question grotesque. Ne faudrait-il pas dire à l'opposé : le théâtre peut-il seulement vivre sans ses auteurs ${ }^{1}$ ?». Lorsque Ulrich Khuon développe, au milieu des années 1990, son concept d'Autorentheater, il ne prône pas un retour à ce que l'on entend, en France, par « théâtre d'auteur » (Literaturtheater), en opposition au Regietheater (dans les excès duquel Klaus Völker voit d'ailleurs le danger d'un «thêâtre d'auteur $»^{2}$ où c'est le metteur en scène qui détient l'autorité), mais la « collaboration continue entre metteur en scène, auteur, comédiens et dramaturge (au sens allemand du terme) $»^{3}$. C'est dans ce contexte expérimental que Dea Loher, née en 1964 à Traunstein (Haute-Bavière), fit ses débuts d' " auteure de pièces de thêâtre »(Dramatikerin) avec Leviathan (1993). Fremdes Haus, qu'elle écrivit ensuite pour le Schauspiel Hannover, marqua en 1995 la naissance du fameux « Dreamteam Loher-Kriegenburg », cette collaboration « entre une auteure et un "faiseur de théâtre" » confirmée avec

1. - Ulrich Khuon, Das Spiel des Schreibenden und seine Anstöße. Dea Loher und das Autorentheater in Hannover, in : Jens Gross/ Ulrich Khuon, Dea Loher und das Schauspiel Hannover, Niedersächsisches Staatstheater Hannover GmbH, 1998, p. 12. Désormais cité sous la forme «Hannover ».

2. - Klaus Völker, Regietheater und Autorentheater, Neue Zürcher Zeitung, 07.07.2013, http://www.nzz.ch/aktuell/startseite/article8V82J-1.274679.

3. - Nicht Harmonisierung, sondern Dissonanz. Juliane Kuhn im Gespräch mit Dea Loher, Hannover, op. cit., p. 21. 
Das letzte Feuer (2008) comme la collaboration entre « partenaires à égalité », puisque l'auteure obtint le « Mülheimer Dramatikerpreis » pour la pièce et Andreas Kriegenburg le « Faust-Theaterpreis » pour sa mise en scène au Thalia Theater de Hambourg. Dea Loher n'écrit pas pour le théâtre dans le sens où elle formulerait en vue de la mise en scène, mais elle met le texte à disposition ${ }^{4}$. Le terme de « faiseur de théâtre » (Theatermacher) renvoie alors à la mise au jour par le metteur en scène de la théâtralité5 inhérente au texte de théâtre. Sachant qu'elle est susceptible aussi bien d'improviser un texte dans un travail collectif avec les comédiens (Blaubart - Hoffnung der Frauen à Munich en 1997) que d'interdire une mise en scène lorsqu'elle estime sa pièce mutilée (en 2013, lorsqu'Alexander Riemenschneider supprima un personnage de Unschuld), que signifie : mettre le texte à disposition ? Livrer au metteur en scène un « matériau ${ }^{6}$, cela a-t-il le même sens pour Dea Loher que pour Heiner Müller ?

Comme son titre le laisse entendre, Verkommenes Ufer Medeamaterial Landschaft mit Argonauten ${ }^{7}$ est la juxtaposition de trois textes autonomes, les concepts de « paysage » et de «matériau » primant sur celui de personnage. Le texte de Müller, dont la genèse s'étend sur plusieurs décennies, porte les traces de la mutation du dialogue dramatique au cours du $\mathrm{xx}^{\mathrm{e}}$ siècle, son écrasement progressif par le monologue, lequel glisse lui-même vers la «monologie ». Le texte central recèle des vestiges du dialogue traditionnel : un long monologue de Médée subvertit le système de la communication interpersonnelle. Un flux de paroles sans destinateur ni destinataire investit l'espace du troisième texte dont le lien avec Jason passe par les voies encore inexplorées du rêve et de l'imaginaire collectif. Le premier texte mêle des voix multiples, bribes de conversations triviales de sources diverses non marquées. Avec Manhattan Medea, qu'elle écrivit à l'aube du XXI ${ }^{\mathrm{e}}$ siècle (1999) pour le festival d'avant-garde « Steirischer Herbst », et qui fut créé à Graz par Ernst M. Binder en collaboration avec le

4. - « Für mich ist Dea Loher augenblicklich die beste deutsche Feder. Sie ist schwierig, da sie nicht auf das Theater hin formuliert, sondern den Text zur Verfügung stellt und sagt: "geht damit um, er ist da" », Kargheit, Lust, Trauer, Liebe, Sehnsucht, Zweifel. Kekke Schmidt im Gespräch mit Andreas Kriegenburg, Hannover, op. cit., p. 123.

5. - Gerda Poschmann, Der nicht mehr dramatische Theatertext. Aktuelle Bühnenstücke und ihre dramaturgische Analyse, Tübingen, Niemeyer, 1997. Poschmann fait de ce concept un critère positif pour l'analyse des textes de théâtre «non dramatiques ».

6. - Selon Dea Loher, les monologues sont un «matériau » que l'on peut traiter librement, Kekke Schmidt im Gespräch mit Andreas Kriegenburg, op. cit., p. 125.

7. - Heiner Müller, Werke 5 : Die Stücke 3, Francfort-sur-le-Main, Suhrkamp, 2002, p. 71-84. 
Mecklenburgisches Staatstheater Schwerin ${ }^{8}$, Dea Loher contribue-telle à la « renaissance du drame dramatique $»^{9}$ en réintroduisant ses éléments structurels, le personnage, l'action et le dialogue, abandonnés par Heiner Müller pratiquement deux décennies plus tôt, aux grandes heures du «théâtre postdramatique »? Ou, à l'époque où Müller est lui-même revenu, avec Wolokolamsker Chaussee, à une forme dramatique plus immédiatement compréhensible par le plus grand nombre ${ }^{10}$, contribue-t-elle à l'émergence de ce « texte de théâtre » qu'il appelle de ses vœux lorsqu'il déplore qu' " au théâtre le temps du texte n'est pas encore venu ${ }^{11}$ ?

Le titre Manhattan Medea signale bien le retour du personnage, son association à une ville symbole du capitalisme justifie toutefois la question du rapport entre fiction intra-scénique et « paysage » (urbain/ social). En actualisant la tragédie d'Euripide, Dea Loher met à l'épreuve le drame, dans une forme plus souple que le drame aristotélicien au sens strict. Les personnages nouveaux sont dotés d'une double fonction. Le portier peintre Velasquez, qui remplace les personnages secondaires de la nourrice et de l'éducateur, allie la fonction intra-scénique de l'observateur, informateur, intermédiaire du portier à la fonction extrascénique, autoréférentielle, du peintre. Le « travesti sourd » Deaf Daisy, inventé de toutes pièces pour pallier la perte des pouvoirs magiques de Médée, allie de la même façon à sa fonction d'adjuvant selon le schéma actanciel une fonction auto-thématique et métathéâtrale. À côté des scènes dialoguées - Médée et Velasquez (tableau 1), Médée et Jason (2 et 8), Médée et Deaf Daisy (4 et 7), Médée et SweatshopBoss (5) -, on observe la présence d'un espace extra-scénique, un « hors scène » multiforme. L'abandon de l'axe intra-scénique (interne) de la communication au profit de l'axe externe (du theatron) étant, selon Hans-Thies Lehmann, la propriété essentielle du « théâtre postdramatique », il semble judicieux d'examiner Manhattan Medea sous

8. - Dea Loher, Manhattan Medea. Blaubart - Hoffnung der Frauen, Francfort sur le Main, Verlag der Autoren, 1999 ; Barbe Bleue, espoir des femmes. Manhattan Medea, traduit de l'allemand par Laurent Muhleisen, Paris, L'Arche Éditeur, 2001. Nous nous référons implicitement à cette traduction. Tous les textes de Loher sont édités au Verlag der Autoren, nous citerons implicitement d'après cette édition (seule indication de la page).

9. - «Die Renaissance des dramatischen Dramas », in : Birgit Haas, Plädoyer für ein dramatisches Drama, Vienne, Passagen Verlag, 2007, p. 177. Haas fait de Dea Loher le fer de lance de son plaidoyer pour le « drame dramatique ».

10. - Heiner Müller, Werke 5, op. cit., p. 215-222. Voir Francine Maier-Schaeffer, «"Stücke lesen sich anders zu andrer Zeit". Heiner Müller und der 17. Juni 1953 », in : Études Germaniques 68 (2013), 2, p. 268-275.

11. - Theresia Birkenhauer, « Die Zeit des Textes im Theater», in : Artur Pelka/ Stefan Tigges (Hg.), Dramatische Transformationen. Verwandlungen dramatischer Formen in Deutschland seit 1945, Bielefeld, Transcript Verlag, 2011, p. 247-261. 
l'angle de la double adresse du texte de théâtre, si l'on veut mesurer son degré d'émancipation de la forme traditionnelle. Nous distinguerons deux voies pour la communication sur l'axe externe du système, la voie directe qui, au sein du dialogue, se superpose à l'axe intra-scénique et la voie qui passe par un réseau de relations inter-textuelles (au sein de l'espace constitué par les scènes dialoguées et le « hors scène »).

\section{Un autre dialogue}

Au début du dialogue (tableau 2), le spectateur n'a pas encore atteint le niveau d'information des personnages : s'il a appris dans le premier tableau, qui tient lieu de prologue, que Médée vient à la demande de Jason, il ne connaît pas le contenu de la lettre qu'elle a reçue. Il ne découvrira que tardivement l'objectif de la rencontre. Le dialogue, selon Hegel « la forme dramatique par excellence » qui fait progresser l'action vers la résolution du conflit, est sans cesse contrarié par un deuxième mode de communication, qui emprunte les voies épiques du récit et de la description. Les stratégies bien connues de la querelle conjugale, le rappel de la première rencontre et des sacrifices consentis, orientent le dialogue vers le passé et la relecture de l'histoire.

Dea Loher dote Jason d'un passé antérieur à la rencontre de Médée et fait ainsi de lui un personnage proche de ceux qui peuplent ses propres pièces, notamment Jane qui, dans Fremdes Haus (1995), est coupable, mais aussi victime - victime de la guerre, de la guerre des Balkans que Jason a fuie en sacrifiant sa mère. L'événement traumatique et la confidence faite à Médée sont à l'origine de leur histoire commune. Les souvenirs qui se rattachent à sa mère, le récit de sa mort, entrecoupé de longs silences, dont le rythme contraste avec la rapidité du dialogue, est un langage que Médée ne veut entendre. La femme bafouée fait à la lumière du présent une relecture destructrice du passé. En cinq phrases rythmées par la répétition de leur amorce « Aujourd'hui je pense », elle transforme une histoire d'amour en une chaîne de trahisons. Jason avoue «froidement » (les seules didascalies du dialogue le précisent ${ }^{12}$ ) avoir « laissé mourir » sa mère, comme il avouera qu'il n'a jamais voulu rester avec Médée et qu'il continuera à la tromper jusqu'à ce que cette vie vaille la peine d'être vécue. Il avait besoin d'argent pour se réfugier en Amérique, il a besoin d'argent pour y vivre, ce sont « les circonstances », « la nécessité », qui décident. La sincérité de son pragmatisme se heurte à la radicalité de la justicière tragique.

12. - Loher fait un usage très parcimonieux des didascalies pour laisser toute liberté au metteur en scène. Ici, l'indication scénique est exceptionnellement longue et précise, p. 27. 
Le sacrifice de Médée est le meurtre de son frère, commis avec la complicité de Jason sur le bateau qui les menait en Amérique après qu'ils eurent volé à son père une maigre «toison d'or » ne permettant pas de nourrir en plus des trois passagers clandestins l'enfant à naître. Elle fait de la culpabilité partagée un argument contre leur séparation. La langue montre dans sa matérialité le refus de la remémoration : « Non/Non/Non je ne pense pas à cela », « Tais-toi [...]/Tais-toi [...]/Tais-toi sorcière », « Pour toi pour toi/Moi Un meurtrier Moi Un meurtrier/ Toi Sorcière ». Les sonorités allemandes de Dich/Ich et Hexe sont encore plus «parlantes » ${ }^{13}$. Sous la pression de Médée, qui exhibe l'arme du crime et s'entaille la main puis le visage, Jason confie la vision qui le hante : «Parfois, je crois/ voir les yeux de ton frère dans l'enfant $»^{14}$. Prisonnière de sa passion, Médée n'entend pas la langue du traumatisme. Le dialogue est pour Jason une maïeutique. Il découvre dans la douleur ses contradictions, la communication impossible et les pièges du langage.

Le spectateur familier du mythe est susceptible d'identifier le moment qui fait progresser l'action, si l'on admet que l'action est le double meurtre :

$\begin{array}{ll}\text { MEDEA } & \text { Ja. Sie ist jung. Sie ist schön. } \\ & \text { Ihre Haut ist wei } \beta \text {. } \\ \text { JASON } & \text { Ja. Ihre Haut ist wei } \beta \text { und - } \\ \text { MEDEA } & \text { Und - } \\ \text { JASON } & \text { Und - } \\ & \text { Sie ist Unschuld. }\end{array}$

Schweigen.

MedEA Geh Jason

und feiere Hochzeit. (p. 37-38)

La motivation sera explicitée dans le deuxième dialogue de Médée avec Deaf Daisy : «L'innocence doit brûler »15. Le meurtre de l'enfant (unique) n'est à aucun moment verbalisé. Le spectateur averti en reconnaîtra le projet dans une réplique du dialogue entre Médée et Sweatshop-Boss, le père de la jeune fiancée ${ }^{16}$.

13. — «Nein/Nein/Nein daran denke ich nicht », « Schweig [...]/Schweig [...]/ Schweig Hexe », « Für dich für dich/ Ich Ein Mörder Ich ein Mörder/ Du Hexe/ », p. 30 $s q$.

14. - «Manchmal glaube ich/ die Augen deines Bruders in dem Kind zu sehen. $[\ldots] »$, p. 34 .

15. - « Die Unschuld soll brennen », p. 54.

16. - « Ich habe ein Leben für ein anderes genommen. Meinen Bruder, der wie mein zweites Leben war. Um eines Mannes willen, der kein Messer wert ist. Jetzt zählt 
Manifestement, la vocation du dialogue n'est pas de faire progresser l'action. Le dialogue argumentatif se mue en dialogue d'investigation. «Le théâtre est pour moi l'espace de la langue »17, affirme Dea Loher. L'attention va en effet davantage à la langue et, davantage qu'au texte, au « subtexte », ce langage non verbal - tirets, pauses, espaces, bégaiements et blocages - logé dans la matérialité du texte à destination du lecteur/ auditeur. Le rythme du texte est lisible dans l'écriture comme une musique : répétition de mots brefs (comme une succession de croches) complétés à la troisième occurrence par une période plus longue, répétition de couples de mots sans ponctuation, espaces typographiques plus ou moins grands. Ce dialogue révèle les limites de la communication interpersonnelle, la résistance des personnages et l'insuffisance des mots, donc les limites de la «mimesis en action » qu'est pour Aristote le dialogue dramatique.

\section{Hybridation et épicisation}

Forme transitoire entre le dialogue épicisé que nous venons de voir et le « hors-scène » absolu, le tableau 8 n'est pas sans rappeler la structure de Medeamaterial de Müller. Le deuxième dialogue avec Jason est en effet précédé d'un "monologue » de Médée, composé d'un texte en vers libres de huit lignes, la répétition de la vision de Jason qu'elle s'approprie ${ }^{18}$, à la différence près qu' elle s'adresse à son enfant (absent), et d'un long texte en prose, récit détaillé du traumatisme dont Jason a refusé de se souvenir. La particularité de ce second texte réside dans le fait que les paroles prononcées sur le bateau dans la nuit du meurtre sont rapportées au discours direct. Selon Aristote, la mimesis épique se distingue de la mimesis dramatique par l'introduction d'un narrateur qui crée la distance. Ici, il n'y a pas de distance entre le narrateur et le personnage principal, le récit est à la première personne, le temps de la narration est le présent. La longueur des paroles reproduites, parfois monosyllabiques, entrecoupées par le bref verbe dire, souvent répété, parfois omis, le redoublement du pronom personnel lorsque le narrateur rapporte ses propres paroles sans verbe introducteur (Moi Moi - la traduction française ne rend pas le redoublement strident Ich Ich), les points qui segmentent les phrases par-delà les règles grammaticales, la rapide alternance des noms de locuteurs, impriment au récit un mouvement autre que celui de la syntaxe, le doublent d'une signification autre

nur noch das Kind. Und dieses Leben, für das ich getötet habe, wirst du mir nicht nehmen. Und keiner jemals », p. 50 sq.

17. - Theater ist für mich der Raum der Sprache. Eva Heldrich im Gespräch mit Dea Loher, Hannover, op. cit., p. 88.

18. - Voir note 14. «Manchmal glaube ich/ die Augen meines Bruders zu sehen/ in den deinen. [...] », p. 56. (Nous soulignons). Notons aussi le rythme brisé. 
que la sémantique, perceptible par les sens. Le dialogue à trois voix en staccato transmet au spectateur la violence de la remémoration. Puis le récit reprend son cours. Outre que la voix du narrateur évoque le frère comme un étranger, la déponctuation totale déstabilise le lecteur/ spectateur :

Der Bruder packt ein Rohr Ich erschlage dein Fleisch Hexe Wenn du es nicht tust Ich greife in den Brotsack und fühle das Messer Jason fällt den Mann von hinten an und fasst seine Arme Meine Hand sticht zu Jason Wir haben den Leib des Mannes in Fetzen aus Plastik gewickelt (57)

Les paroles rapportées, plus longues, à la première personne du singulier, se mêlent à la narration à la même personne sans aucun encodage du discours. Après la phrase en suspens, le nom de Jason déroute : le lecteur ne comprend pas d'emblée que la dernière partie du récit, à la première personne du pluriel, est adressée directement au personnage absent.

La construction de ce texte ressemble fort à l'écriture de Heiner Müller qui marque son retour au Lehrstück brechtien dans la deuxième moitié des années 1980. Dans Das Duell (Wolokolamsker Chaussee III), le flux de conscience d'un protagoniste à la première personne fait remonter à la surface les couches enfouies dans la mémoire sous la forme de paroles rapportées au discours direct. La déstabilisation du lecteur provient, comme chez Dea Loher, de l'absence de répartition des répliques non attribuées à un locuteur, juxtaposées sans encodage. Müller puisa dans le fragment Fatzer, dont il fit à son tour le laboratoire des formes dramatique et épique qu'il fut pour Brecht entre 1926 et 1930 , les deux techniques formelles qui sont aujourd'hui également la marque de fabrique des textes de Dea Loher, l'épicisation du dialogue (lequel est souvent le découpage d'un récit préalablement écrit en prose) et la langue brisée (le «Fatzervers ${ }^{19}$ ). Dans le prologue de son adaptation de l'Antigone de Sophocle (1948), Brecht « narrativise » le dialogue. Le récit est réparti entre les deux personnages (Sœur I et Sœur II) qui mêlent à la narration le dialogue dramatique ${ }^{20}$. Dans le « livre de modèle d'Antigone », Brecht précise que les phrases au passé doivent être adressées au public, tandis que les dialogues doivent être

19. - Bertolt Brecht, Journal, 10 janvier 1951. Il s'agit des vers libres, avec notamment des enjambements qui brisent la syntaxe. Voir Francine Maier-Schaeffer, Les Métamorphoses du dieu Bonheur. Heiner Müller, Bertolt Brecht et l'"écriture de fragment », Paris, PUPS, 2013, p. 137-159.

20. - Antigone, in : Bertolt Brecht, Werke. Große kommentierte Berliner und Frankfurter Ausgabe, Bd. 8, Stücke 8, Berlin et Weimar, Aufbau Verlag/ Francfort sur le Main, Suhrkamp, 1992, p. 195-199. 
joués avec les déplacements et les gestes ${ }^{21}$. C'est une leçon pratique du jeu épique qui se meut alternativement sur les deux axes, intra-scénique et extra-scénique. Brecht inscrit dans le texte le procédé de répétition qui consiste à mettre le comédien dans la situation du narrateur en transposant le texte à la troisième personne pour faciliter le jeu distancié22. À la différence de Der gute Mensch von Sezuan (considéré comme un cas d'école du théâtre épique) où, au tableau 8 , le récit de l'action passée, adresse frontale au public par un personnage qui sort de son rôle, alterne avec sa représentation, l'illusion de la «mimesis en action »n'est pas sans cesse interrompue, elle est d'emblée empêchée. Le spectateur n'est pas exhorté à réagir, il est déstabilisé (verfremdet au vrai sens du terme) par la distanciation inscrite dans l'écriture.

Dea Loher expérimente de multiples manières l'inscription de la distanciation dans le texte. Ainsi, Linda, un personnage de Diebe, raconte (au passé), dans un très long monologue à la troisième personne, une expérience vécue qu'elle commente à l'adresse d'un mari et d'un enfant qu'elle n'a pas, mêlant au récit le discours rapporté (10). Pour la création de la pièce, en 2010, au Deutsches Theater de Berlin, Andreas Kriegenburg mit en œuvre le procédé consigné par Brecht en faisant jouer les didascalies intégrées au discours rapporté : «Les figures disent leur dialogue et thématisent de façon épique (narrative) leur statut de personnage et jouent de surcroît leur texte ${ }^{23}$. Le comique mis au jour par le jeu épique est également sous-jacent dans le staccato de la discussion rapportée. Un metteur en scène pourrait choisir de le libérer en représentant, par exemple, la succession des paroles échangées comme un numéro de slapstick chaplinesque. Les personnages perdent alors leur réalité pour devenir des marionnettes. La langue devient première, matériau mis à disposition.

Le texte en prose en deux parties peut avoir, sur le plan intrascénique, la fonction de monologue intérieur de Médée, seule en scène avant l'arrivée de Jason. La didascalie «Entre Jason » indique le changement de registre et le retour au dialogue traditionnel. Ce tableau est-il alors comparable à Medeamaterial de Müller? Le dialogue est en effet concurrencé par le monologue, sa dramatisation (par le discours rapporté) transforme le monologue en une scène de " théâtre dans le théâtre » intérieur d'un personnage enfermé dans ses visions ; après la «monologie », le dialogue intra-scénique reprend certes, mais c'est un dialogue rétréci, car Jason a recours au récit d'un rêve ${ }^{24}$ pour exprimer

21. - Antigonemodell 1948, ibid., Bd. 25 : Schriften 5, 1994, p. 84-89.

22. - Ibid., p. 79.

23. - Elena Philipp, « Da hab' ich gemerkt, dass ich kein Individuum bin », www. nachtkritik.de.

24. - Ce rêve est très explicite, contrairement aux rêves (effectifs ou éveillés) 
sa solitude et son déchirement, un dialogue troué de deux longs silences, un dialogue dramatique qui scelle son échec.

Dans l'œuvre ultérieure, les deux registres fusionnent, mêlant le dit et le pensé, l'imaginaire et le vécu, la vie ordinaire et la vie rêvée. Dans Das Leben auf der Plaça Roosevelt (2004), par exemple, une réplique du dialogue entre Linda et Punto surprend le lecteur, puis la didascalie « À Punto » achève de le déstabiliser ; il apprendra par une confidence dans un autre dialogue que la réplique en question dit les paroles que la secrétaire aurait voulu dire à son patron (123). Dans Unschuld (2003), le personnage sans nom interrompt sa réplique : «C'est ce que j'aurais aimé lui dire, mais je ne le fis pas dans la réalité, seulement dans ma tête. Dans la réalité je lui offris une tasse de thé ${ }^{25}$, et il corrige sa réplique en intégrant les didascalies aux paroles rapportées au discours direct. La «mimesis en action » est démasquée comme l'illusion de la réalité par la liberté laissée au personnage de jouer son rôle de comédien (d'imiter) ou de sortir de son rôle (d'inventer), ce qui revient à superposer deux illusions. Le lecteur ne s'aperçoit que le personnage a quitté son rôle que lorsqu'il le réintègre. Le « théâtre dans le théâtre » du dialogue loherien, qui jette le soupçon sur le dialogue dramatique, met aussi le théâtre à l'épreuve.

\section{Le « hors scène »- théâtre des voix}

Le « monologue » de Médée appartient également au « hors scène » composé de ces autres textes qui racontent l'histoire par d'autres moyens que le dialogue et l'articulation verbale. Sans lien apparent avec la fable, un texte dont la forme est celle du poème en prose occupe seul l'espace de la page (tableau 3). On peut y voir une réminiscence de l'espace réservé, dans la tragédie antique, au Chœur, où Médée confie au Coryphée ses sentiments et ses desseins ${ }^{26}$. Ici, seule la voix de Médée est présente, elle n'est pas adressée, le texte d'un haut degré de poéticité n'est pas immédiatement compréhensible. Le poème est

du texte de Müller. Voir « Episierung des Dramas? Der "Ich-Monolog" Landschaft mit Argonauten zwischen epischem Theater und (post)moderner écriture », in : Ian Wallace / Tate Dennis / Gerd Labroisse (Hg.), Heiner Müller: Probleme und Perspektiven. BathSymposium 1998, Amsterdamer Beiträge zur neueren Germanistik, Amsterdam, Rodopi, 2000, p. 237-257.

25. - «Das hätte ich ihr alles gerne gesagt, aber ich tat es in der Wirklichkeit nicht, nur in meinem Kopf. In der Wirklichkeit bot ich ihr eine Tasse Tee an [...] », in : Unschuld, p. 22.

26. - Chez Euripide, l'intérieur de la maison est réservé aux lamentations de Médée et au meurtre de ses enfants, les dialogues ont lieu sur le seuil. Chez Loher, le lieu des dialogues est l'entrée de l'immeuble de la $5^{\mathrm{e}}$ avenue de New York appartenant au futur beau-père de Jason qui y est déjà installé. 
redonné au tableau 6, fragmenté et troué. Seule la strophe centrale demeure intacte :

Nicht für Jason

Nicht für mich

Nicht für das Kind

Nicht weil das Messer in meiner Hand Weil das Messer in meiner Hand war

Für ein Gesetz

Der Falke

Lilie im Schnabel

Fliegt über Schnee (40)
Für Jason

Für das Kind

war

Für welches Gesetz

Der Falke

Lilie im Schnabel

Fliegt über Schnee (53)

La troisième strophe est entièrement nouvelle :

Falke ohne Lilie

Bleibt

Roter Schnee (53)

Le faucon symbolise la liberté dans la mythologie de la Macédoine où Dea Loher a séjourné avant d'écrire Fremdes Haus. La pièce contient trois longs monologues dont le dernier est «Le rêve du faucon ». Le motif du faucon tisse un lien avec Jason et avec la chanson populaire macédonienne que chante Deaf Daisy. Les paroles que Médée adresse à son frère à travers son enfant qu'elle s'apprête à étouffer (tableau 10) sont la dernière étape de l'histoire racontée par les deux versions du poème et le monologue intérieur :

Nur für mich.

Für mich.

Für mich.

Schweigen.

Ich liebe dich. (61)

La poésie est l'espace dans lequel le tragique, l'indicible, l'histoire intime, se retire. Peut-être faut-il simplement écouter la voix, comme le suggère Deaf Daisy, qui ne connaît pas sa propre voix, mais qui sent ses pulsations ${ }^{27}$. Médée est la « sœur » du «travesti sourd ( « ni homme ni femme », selon le désir de la Médée de Müller dans Medeamaterial). À

27. - «Ich kenne nicht meine eigene Stimme. Aber ich fühle sie pulsieren. », p. 43. 
la manière d'un thème musical, une chanson l'accompagne par bribes, thème de la colombe et de la mort, qui devient aussi le thème de Médée dans la musique de la pièce, et qui se déploie en entier dans le tableau final. Elle accompagne la question prémonitoire de Deaf Daisy, le sourd qui entend le silence, le silence de la ville, et le silence qui précède la mort :
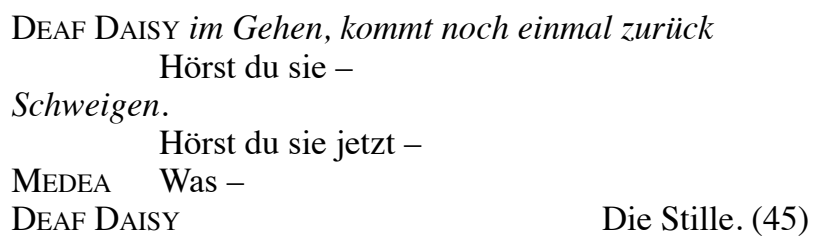

On peut imaginer qu'un metteur en scène opte pour la version originale de la « très vieille chanson macédonienne » (dont le texte est donné en note de bas de page, p. 92). Dans Diebe, le pouvoir de la chanson, des sonorités et du rythme qui transcendent le logos, est à la fois démontré et thématisé. La chanson (message d'un mort) a une fonction intra-scénique (de compréhension de l'incompréhensible) et « inter-scénique » (de reconnaissance) ${ }^{28}$; sur l'axe extra-scénique, le dialogue invite à écouter, dans le théâtre de Loher, la musique de la langue, les silences, les vides et les creux, le langage non verbal du « subtexte » :

LiNDA Hören Sie denn nicht, wie das klingt. Hören Sie zu, hören Sie, wie das klingt... wiederholt ein paar Worte... Das könnte man singen.

Pause

RAINER Ja. Sie haben Recht. Das kann ich mir vorstellen. Wenn Sie das täten, wenn Sie diese Worte, die unbekannten spanischen Worte singen würden, dann würden sie ein Lied. - Ja doch, jetzt höre ich es auch. Ich höre es.

Lied (92)

\section{Dialogue métathéâtral}

Le dernier tableau, le dénouement, réunit le double niveau intrascénique, avec le double meurtre, et extra-scénique, avec le double thème autoréflexif et métathéâtral qui traverse l'œuvre : celui de la représentabilité de la réalité par l'artiste, amorcé dès le prologue avec

28. - C'est grâce à des bribes de cette chanson, qui accompagne Ira comme un thème musical (p. 59, p. 81), que le spectateur pourra, à l'insu des personnages, établir un lien entre des êtres en souffrance qui se cherchent et se croisent sans se (re)connaître. 
le personnage du peintre Velasquez, et celui du spectacle, développé dans le dialogue entre Médée et Deaf Daisy. Sur le plan intra-scénique, Velasquez, chargé de surveiller la rencontre entre Médée et son enfant, lui offre en gage de son dévouement une copie de l'Infant Philippe Prosper de Diego Velasquez (1659), puis les laisse seuls, permettant ainsi au destin (de la tragédie grecque) de s'accomplir. L'infanticide se réduit à une indication scénique, le meurtre de la jeune fiancée est absorbé par le spectacle. Selon la didascalie « apparaît une torche ardente ", elle embrase le tableau de Velasquez, Deaf Daisy chante la chanson populaire macédonienne, lorsque la flamme et la voix s'éteignent simultanément, apparaît le tableau de Picasso Las Meniñas. Cette apothéose finale fait du feu d'artifice que Médée offre à Deaf Daisy en échange de la robe toxique une métaphore de la création. Le spectacle écrase la tragédie comme le tableau cubiste supplante la copie du portrait classique. "La jouissance de la destruction et de la création, qui n'est parfois que la pure jouissance de la vie », est aux yeux de Dea Loher une des légitimations de l'art ${ }^{29}$. Le drame brûle-t-il ?

La peinture offre à Dea Loher la possibilité d'observer sa propre écriture à partir de la distance d'un autre medium. La peinture et l'écriture ont en commun, la première depuis l'apparition de la photographie, la seconde depuis la révolution industrielle, d'être confrontées au problème de la représentabilité de la réalité par l'art avec la prétention de penser la réalité comme transformable avec les moyens de l'art. Pour cette raison, elle cherche le plus souvent, lorsqu'elle commence à écrire une pièce, une œuvre dans le domaine de la peinture susceptible de servir de pendant à son sujet ${ }^{30}$. Pour la réécriture de la tragédie d'Euripide, le parallèle avec la peinture, où la copie des maîtres est pour ainsi dire institutionnalisée, s'est sans doute imposé de lui-même pour questionner le rapport entre répétition et innovation. L'admiration de Picasso pour Velasquez, dont il a copié de nombreuses œuvres, notamment l'énigmatique Las Meniñas, a certainement motivé le choix du peintre de cour espagnol pour doubler la fonction intra-scénique du portier. Le lien de Picasso avec la question de la représentabilité de la réalité et de l'engagement de l'art n'a pas besoin d'être explicité, il suffit de penser à Guernica. Il n'est pas précisé laquelle des quarante-quatre études de Las Meniñas doit remplacer le portrait de l'infant Philippe

29. - Spectaculum 76. Sechs Moderne Theaterstücke, Francfort-sur-le-Main, Suhrkamp, 2005, p. 269.

30. - Dea Loher, Rede zur Verleihung des Gerrit-Engelke-Preises, Hannover, op. cit.,p. 224. Dans ce discours, elle commente la peinture de Richter. Pour Fremdes Haus, les tableaux de Lucian Freud l'ont accompagnée, ainsi qu'une icône d'une « minuscule église macédonienne », ibid., p. 117. Dans Land ohne Worte (2007), elle passe par le peintre Mark Rothko, fondateur de l'« expressionnisme abstrait », pour exprimer ses propres difficultés à trouver les mots adéquats après son retour de Kaboul. 
Prosper, le tableau dans sa totalité 31 ou plutôt l'une des nombreuses variations de l'infante Marguerite qui donnerait à voir, à l'exemple d'un autre portrait d'enfant, une copie différente de celle du Velasquez fictif, une copie expérimentale qui transgresse les normes, qui invente de nouvelles formes, une copie qui devient création par transformation de l'original. Le tableau de Picasso réalise en effet le programme que Velasquez énonce sans parvenir à le concrétiser :

Schön - ich rede von der Malerei, nicht von Werbung. Nicht von der Oberfläche, sondern wie sich diese Oberfläche zusammensetzt. Das, was dem ersten Eindruck widerspricht, was sich dem Schein entgegenstellt, zum Beispiel, das macht Schönheit. (15)

Déconstruire afin de voir, puis donner (et apprendre) à voir « comment cette surface s'agence », c'est le programme du cubisme qui fracture et démultiplie les perspectives. Montrer les choses non pas comme on les voit (par le fait de l'habitude), mais comme elles sont vraiment, en profondeur, c'est aussi le programme de Brecht, pour lequel il élabora le fameux « effet de distanciation ». La réécriture de la tragédie d'Euripide est une manière de concrétisation de ce programme : le dialogue entre Médée et Jason, dont la violence est inscrite dans le corps des mots et le corps de la page, construit, pour le spectateur qui entend le « subtexte », le Jason qu'avec ses yeux de peintre le portier Velasquez devine sous le costume taillé sur mesure et qui néanmoins fait des plis au niveau des épaules, à cause de la démarche voûtée de l'homme malheureux (15). Ce portrait émerge du costume du mythe et de ses réécritures qui le donnent à voir exclusivement à travers les yeux de Médée et des femmes du Chœur d'Euripide. Les propos de SweatshopBoss ajoutent une perspective complémentaire (47).

La démythification de la tragédie est un spectacle à l'invention duquel Dea Loher associe le spectateur. La Médée moderne ayant perdu ses talents de « sorcière », elle a besoin d'aide pour mener la tragédie à son dénouement. Le public assiste pour ainsi dire en temps réel à la création de l'adjuvant Deaf Daisy. Lorsque le «travesti sourd » entend le nom de Médée, il « siffle doucement ». Il montre ainsi qu'il connaît le mythe et comprend le rôle qui lui est attribué. Le public, également familier du mythe, devient complice de la « copie». « Disons, je donne ce sac-poubelle pour un spectacle. Ce sera une comédie, ce sera une tragédie, aucune importance tant que c'est une véritable pièce ${ }^{32}$, cela

31. - Les correspondances entre la pièce de Loher et le tableau dont la description ouvre Les Mots et les Choses de Michel Foucault sont nombreuses. Leur analyse dépasserait le cadre de cette contribution.

32. - « Sagen wir, ich gebe diesen Müllsack für ein Schauspiel. Wird es eine Komödie, wird es eine Tragödie, es tut nichts, wenn es nur ein echtes Spiel ist. », p. 54. 
signifie : je joue le jeu, pour que la tragédie ait lieu, mais ce ne sera pas la tragédie d'Euripide, ce sera " autre chose ». Le personnage créé par Loher n'est pas un personnage comme les autres. Il est le double maléfique de Médée, une figure étrange, qui « danse avec la mort », cynique, qui voit dans la ville une Sodome dont les anges déchus doivent être brûlés. Ce personnage irréel s'associe à Médée pour faire de la mort de sa rivale un spectacle aussi grandiose que les feux d'artifice de la fête de l'Indépendance. Aussi ambivalent que le feu, à la fois destructeur et régénérateur, il commente ses propres visions apocalyptiques : «Pauvres de nous ${ }^{33}$.

L'importance accordée à l'authenticité de la pièce - ou plutôt du jeu, du spectacle - souligne le caractère artificiel de l'art. L'art n'est pas la vie, le feu sera aussi artificiel que les feux d'artifice, aussi artificiel que le moyen trouvé pour tuer la fiancée, aussi artificiel que le personnage créé à cet effet. Le spectateur n'a aucune raison de s'identifier et de "souffrir avec ", il peut jouir de la fête. Si Dea Loher joue avec Medeamaterial, où la Médée de Müller veut transformer sa rivale en « torche de noces » pour offrir une comédie à ses enfants ${ }^{34}$, l'allusion à la pièce de Thomas Bernhard Ist es eine Tragödie? Ist es eine Komödie?, laquelle donne à entendre que la réponse dépend du spectateur, ne peut passer inaperçue ${ }^{35}$. Le spectacle (tragédie ou comédie) comme le tableau (la beauté) sont l'œuvre du spectateur (et du metteur en scène). La remarque de Deaf Daisy est une invite à déceler dans la pièce la connivence de l'auteure avec le public. Son écriture est un jeu avec les modèles, avec le drame traditionnel et ses réécritures. Les éléments comiques ne manquent pas pour celui qui sait (et veut) les déceler : le comique de situation proche du quiproquo dans le prologue (Velasquez refuse de se faire payer pour son rôle d'adjuvant lorsqu'il découvre l'identité de l'inconnue), le traitement parodique du personnage de Sweatshop-Boss, le roi du bizness qui joue au roi Créon (« Tu devras quitter cette ville. Et ce pays [...]. Tu devras le quitter. Sans ton enfant. Sans condition. Parce que je le veux ${ }^{36}$ ). Des répliques telles que «Bon. Ton nom est Médée. Ça signifie quelque chose » et «D'autres que vous m'ont menacée ${ }^{37}$ sont autant de clins d'œil métadramatiques qui font participer le lecteur/ spectateur au plaisir

33. - «Wie arm sind wir. », p. 55.

34. - « Ich will die Braut zur Hochzeitsfackel machen./ [...] Mein Schauspiel ist eine Komödie lacht ihr », op. cit., p. 78.

35. - Birgit Haas, Das Theater von Dea Loher : Brecht und ( $k$ )ein Ende, Bielefeld, Aisthesis Verlag, 2006, p. 265.

36. - « Du wirst die Stadt verlassen müssen. Und dieses Land [...]. Du wirst es verlassen müssen. Ohne dein Kind. Ohne Bedingung. Weil ich es so will. », p. 50.

37. - «Gut. Dein Name ist Medea. Bedeutet das etwas. » (selon l'intonation, le sens de la phrase change), p. 46 ; « Mir haben andere als Sie gedroht. », p. 50. 
de la réécriture. Cependant, la remarque de Deaf Daisy : un « voleur sur sa propriété peut facilement être retrouvé les doigts brisés et les oreilles tranchées - pas que j'attache de l'importance aux oreilles » n'est pas uniquement une plaisanterie. Le « travesti sourd » est également un mendiant faisant les poubelles dans l'immeuble cossu du tyran moderne qui, comme son nom l'indique, a un pouvoir absolu sur les immigrés (clandestins) dont font partie Jason et Médée. La comédie est la forme réaliste de la tragédie du quotidien qui naît des cendres de la tragédie antique. Dès le début, Loher apprécie dans le travail théâtral de Kriegenburg qu'il «talonne la comédie » ${ }^{38}$. À la quête de la perfection formelle, qui condamne le portier Velasquez à rester un « copiste » du Velasquez historique, comme il condamne le peintre Conti, dans le prologue d'Emilia Galotti de Lessing, à demeurer « un artiste sans œuvre » car leurs mains ne sont pas capables de peindre ce que leurs yeux voient, à ce désir d'une perfection impossible, Deaf Daisy oppose la conception que l'on peut qualifier d'auctoriale : «La beauté est le mensonge dans l'œil du spectateur. Célébrons l'imperfection comme belle $\gg^{39}$.

\section{«Au théâtre, la langue doit créer les personnages »}

Manhattan Medea est une pièce de transition entre les premiers succès de Hanovre et les pièces dites de la maturité. Manifestement Dea Loher tire le meilleur parti des stratégies du drame traditionnel (jusqu'au respect de l'unité de temps et de lieu de la tragédie), à l'instar d'un peintre cubiste qui tient à prouver sa parfaite maîtrise des techniques figuratives qu'il laisse derrière lui. Simultanément, elle tire le meilleur parti du jeu avec la double destination du texte théâtral. Le double niveau est une constante de son écriture. Chaque détail est parfaitement motivé sur les plans intra- et inter-scéniques, il appartient au lecteur/ metteur en scène de saisir la superposition des niveaux. Si ses textes ne sont pas mis à disposition de la même manière que ceux de Müller, qui peuvent être utilisés par fragments et recombinés entre eux et avec d'autres ${ }^{40}$, cela tient au fait que Dea Loher conserve les éléments structurels du drame, même éclatés et réduits à leur plus simple expression. La structure de ses pièces n'est pas la fable linéaire avec le conflit dramatique, mais pas davantage la juxtaposition sans lien du théâtre «postdramatique ». Les images disséminées dans les textes sans locuteurs du triptyque de Müller réintègrent le discours des

38. - Warum die Finnen trinken. Kekke Schmidt im Gespräch mit Dea Loher, Hannover, op. cit., p. 115.

39. - « Schönheit - die Lüge im Auge des Betrachters. Feiern wir das Unvollkommene als schön. », p. 55.

40. - Citons à titre d'exemple les expérimentations de Heiner Goebbels. 
personnages. Avec Sweatshop-Boss, la pollution industrielle qui, sur le plan intra-scénique, livre la robe empoisonnée contribue au «paysage » contemporain au même titre que la misère des faubourgs dépeinte par Jason ou le drame familial auquel Deaf Daisy doit sa surdité. Si Dea Loher ne put tolérer la suppression de la « philosophe vieillissante » de Unschuld car « la structure porteuse » et la « cohérence de la pièce » n'étaient plus assurées ${ }^{41}$, c'est parce que cette figure - marginale comme toutes les autres - est « centrale » par son discours : «philosophant » sur les grandes questions de l'existence humaine, elle est aussi investie d'une fonction autoréférentielle ${ }^{42}$. Les personnages de Dea Loher se racontent. Le système de relations inter-scéniques, qui démultiplie les perspectives, tisse un «paysage » de la société avec des figures qui, certes, perdent leur individualité dans un discours épique qui efface l'appartenance d'un texte à un personnage et par conséquent d'un personnage à un acteur, mais qui n'en sont pas moins ancrées dans la réalité de leur temps. La langue crée une atmosphère que le metteur en scène (Theatermacher) doit traduire en théâtre. Le comédien doit renoncer au «parallélisme phono-sémantique » et abandonner « l'intonation qui généralement rend le texte compréhensible $»^{43}$. Dea Loher écrit des « textes de théâtre » (Theatertexte), des textes faits pour le théâtre. La théâtralité du texte et l'oralité de la langue sont à la fois une chance et un défi pour le théâtre. Tout le travail théâtral tient dans cette affirmation : «Au théâtre, c'est la langue qui doit créer les personnages, et non l'inverse ${ }^{44}$.

41. - « Dea Loher sieht "durch die Streichung einer zentralen Figur" sowohl "die tragende Struktur" als auch den "Sinnzusammenhang des Stïcks nicht mehr gegeben" », in : Theaterstreit in Bremen: Autorin untersagt Inszenierung ihres Stückes, www.spiegel. de > Kultur > Gesellschaft > Dea Loher, Montag, 30.09.2013.

42. - À l'instar de Deaf Daisy, elle se prononce pour le « fragmentaire», p. 76.

43. - Olivier Balagna, Orpheus' Blick, Hannover, op. cit., p. 58 sq.

44. - " Auf dem Theater muss die Sprache die Figuren schaffen und nicht umgekehrt », in : Theater ist für mich der Raum der Sprache, op. cit., p. 88. 